\title{
LA CONFÉRENCE DE 1865 ET LE VOLUME DE HETZEL
}

Le 20 janvier 1865, a lieu à la Sorbonne une conférence qui marque une date dans l'histoire de la sémiologie du geste. L'orateur est Pierre Gratiolet. La conférence porte " sur la physionomie en général et en particulier sur la théorie des mouvements d'expression ". Il s'agit pour Gratiolet de populariser des réflexions qu'il mène depuis plus de dix ans, et qui sont en quelque sorte un prolongement de ses travaux de neurophysiologie. Docteur en médecine, mais non praticien, membre fondateur de la Société d'anthropologie de Paris en 1859, longtemps suppléant de son maitre Henri de Blainville pour les cours d'anatomie au Muséum national d'histoire naturelle, sans jamais obtenir la chaire elle-même, Gratiolet est surtout connu pour ses recherches sur le système nerveux et le cerveau, dans une perspective comparative entre l'homme et les primates. Depuis 1863, il occupe la chaire de zoologie de la Faculté des sciences de Paris. La conférence de 1865 marque prématurément la fin de sa carrière puisqu'il meurt moins d'un mois plus tard, le 16 février.

L'année même de sa mort, le texte de la conférence apparait à trois reprises dans des revues et il est publié chez Hetzel avec un texte posthume, De la physionomie et des mouvements d'expression ${ }^{1}$. Ce volume est en quelque sorte une synthèse des conceptions de Gratiolet sur le geste signifiant, et une reprise systématique des réflexions qu'il développait déjà en 1857 dans le deuxième tome de l'Anatomie comparée $d u$ sys-

1. Le texte de la conférence sur la physionomie a été publié :

a) dans Le Moniteur scientifique-Quesneville, $1^{\text {er }}$ février 1865 ,

b) dans la Revue des cours scientifiques du 11 février 1865 ,

c) reproduit dans les Annales de sciences naturelles, $5^{\mathrm{c}}$ série, t. III, Zoologie et Paléontologie, 1865 ,

d) et, enfin, édité chez Hetzel : Pierre Granolet, Conférence sur la physionomie en général et en particulier sur la théorie des mouvements d'expression, suivie de De la physionomie et des mouvements d'expression, suivi d'une notice sur sa vie et ses travaux et de la nomenclature de ses ouvrages, par Louis Grandeau, Paris, Hetzel, 1865.

Revue de synthèse : IV S. $\mathrm{N}^{\text {os }} 3-4$, juil.-déc. 1992. 
tème nerveux ${ }^{2}$. Il s'agit donc d'un travail qui, certes, est en marge de ses intérêts centraux, mais aussi d'un travail de longue haleine et qu'il veut en relation avec ses autres travaux scientifiques ${ }^{3}$.

Le volume publié chez Hetzel a l'intérêt de regrouper deux textes portant sur un même sujet et dont l'un est en quelque sorte la version scientifique, et l'autre la version vulgarisée.

UN ENSEMBLE DE DISCOURS SCIENTIFIQUES ET UN RÉSEAU D'AUTEURS

Par ce glissement d'intérêt vers les gestes d'expression, Gratiolet s'inscrit dans un mouvement qui va se développer au cours de la seconde moitié du $\mathrm{XIX}^{\mathrm{e}}$ siècle, et qui a pour vocation d'élaborer des discours scientifiques sur le geste signifiant, en se démarquant nettement de la tradition physiognomonique et autres courants divinatoires. Cette entreprise de rationalisation est reconnue et développée par tout un ensemble d'auteurs - parmi lesquels Darwin - et pour lesquels Gratiolet est une référence sérieuse. En comparant le jeu des citations mutuelles de ces auteurs, on peut établir que Gratiolet est l'un des plus souvent cités ${ }^{4}$, et l'un de ceux dont le discours est reconnu comme scientifique. Gratiolet lui-même déclare ne pas devoir être confondu avec ceux qui sont tombés dans les « billevesées astrologiques " ${ }^{5}$, ou qui n'ont pas été au-delà d'un art empirique, ou encore, qui ont cru pouvoir déduire un caractère à par-

2. François Leuret, Pierre Gratiolet, Anatomie comparée du système nerveux considéré dans ses rapports avec l'intelligence, Paris, J.B. Baillière et fils, 1839-1857, t. II, 1857. Cet ouvrage est composé de deux tomes dont le premier a été publié en 1839 par François Leuret. François Leuret étant mort, Gratiolet publie seul ce deuxième tome en 1857.

3. On peut noter que dans l'éloge funèbre qu'il fait de Gratiolet, Paul Broca, résumant l'essentiel de la carrière de celui-ci, ne cite pas ses travaux sur la physionomie. Cf. Paul Broca, "Éloge funèbre de Pierre Gratiolet, prononcé sur sa tombe le 16 février 1865 ", Mémoires de la Société d'anthropologie de Paris, 1863-1865, Paris, Victor Masson et fils, 1865 , p. CXII-CXVIII. En revanche, Edmond Alix, dans un discours prononcé en 1867 , évoque assez longuement les travaux de Gratiolet sur la physionomie, et précise que le livre de Gratiolet sur la physionomie était déjà écrit lorsqu'il publie en 1857 le deuxième tome de l'Anatomie comparée: cf. Edmond Alix, Notice sur les travaux anthropologiques de Gratiolet, lue dans la séance solennelle du 20 juin 1867, Paris, Typographe A. Hennuyer, 1869. Rappelons, enfin, que la notice de Louis Grandeau dans le volume de Hetzel, op. cit. supra n. 1 d), compte cinquante-deux références de textes portant pour la plupart sur des questions d'anatomie et de physiologie du système nerveux.

4. L'existence de ce réseau a été mise en évidence in Anne-Marie Drouin, Gestes et physionomie. Problèmes posés par la conceptualisation du geste signifiant. Seconde moitié du xix siècle, thèse pour le Doctorat de l'E.H.E.S.S. sous la dir. de François Bresson, févr. 1989. Voir notamment le tableau p. 224.

5. P. Gratiolet, op. cit. supta n. 1 d), p. 2. 
tir de vagues ressemblances entre certaines physionomies et certains animaux ${ }^{6}$.

DES ANIMAUX, DES HOMMES ET DES HORIZONS THÉORIQUES DIVERGENTS

L'utilisation que fait Gratiolet des références aux animaux n'est pas d'ordre physiognomonique. En cela Gratiolet et Darwin font partie de la même famille de pensée. Pour autant, les références au monde animal ne jouent pas la même fonction chez l'un et l'autre auteur.

Lorsqu'il publie en 1872 The Expression of Emotions in Man and Ani$\mathrm{mals}^{7}$, Darwin montre comment on peut trouver chez l'animal ce que l'on pourrait appeler une étymologie des gestes humains. Autrement dit, ce thème de l'expression des émotions, pour lequel il a certes un intérêt vif et ancien, est pour lui une occasion de fournir des arguments supplémentaires à l'idée d'une transformation des espèces ${ }^{8}$.

Rien de tel chez Gratiolet. Au contraire, comme son maitre Henri de Blainville, Gratiolet interprète la diversité et la parenté des formes vivantes à travers l'idée d'un plan de la création et d'une échelle des êtres, qui n'a rien à voir avec des transformations échelonnées dans le temps ${ }^{9}$.

6. Ibid., p. 4-6 : les auteurs auxquels Gratiolet fait allusion dans la conférence sont surtout Aristote, Della Porta et Lavater. Sur la Physiognomonie, cf. : Jugis Baltrusains, Aberrations. Quatre essais sur la légende des formes, Paris, Olivier Perrin, 1957; François Azouvi, "Remarques sur quelques traités de physiognomonie », Les Études philosophiques, 4, 1978, p. 431-448; Martine Dumont, " Le succès mondain d'une fausse science : la Physiognomonie ", Actes de la recherche en sciences sociales, 54, sept. 1984.

7. Charles DARwin, The Expression of Emotions in Man and Animals, Londres, Murray, 1872, trad. franç. par Samuel Pozzı et René Benoir, L'Expression des émotions chez l'homme et les animaux, $2^{\mathrm{e}}$ éd. Paris, Reinwald, 1890, rééd. Bruxelles, Complexe, 1981.

8. Cf. Yvette Conry, L'Introduction du darwinisme en France, Paris, Vrin, 1974, p. 78, 210, 371 et 378. Cf. aussi Goulven LAURENT, Paléontologie et évolution en France, 1800-1860. De Cuvier-Lamarck à Darwin, Paris, Éd. du Comité des travaux historiques et scientifiques, 1987 , p. 206-207, 222, 254-256. Il faut préciser que, dès 1840, DARwiN avait entrepris une monographie sur l'un de ses fils, observé quotidiennement dans ses expressions et mimiques. On trouve une synthèse de ces observations dans «A Biographical Sketch of an Infant ", Mind, 7, July 1877, p. 285-294.

9. Sur Henri Ducrotoy de Blainville, cf. les articles de Bernard Balan, J. Lessertisseur et F. Joufrroy, Gabriel Gohau, Henri Gouhier, Georges Canguilhem, Revue d'histoire des sciences, t. XXXII, 1, 1979. Cette publication, sous la direction de Bernard Balan, fait suite à une journée d'étude du 16 décembre 1977, en l'honneur du deuxième centenaire de la naissance de Henri Ducrotoy de Blainville. L'article de B. BaLAN, "Organisation, organisme, économie et milieu chez Henri Ducrotoy de Blainville $»$, p. 5-24, cite un passage intéressant d'un article de P. Gratiolet et P. H. E. AlIx, " Recherches sur l'anatomie du Troglydytes aubryi, chimpanzé d'une espèce nouvelle ", Nouvelles archives du Muséum d'histoire naturelle de Paris, Paris, t. 2, 1866, p. 11 : " Les formes extérieures sont la manifestation intelligible et immédiate de la nature de l'animal. Elles racontent sa mission et sa destinée. Elles traduisent par leurs modifications incessantes ses passions et ses instincts, et deviennent par 
L'année précédant la conférence sur la physionomie, il avait fait une conférence du même type à la Sorbonne, intitulée « De l'homme et de sa place dans la création ${ }^{10}$. Il y exposait les résultats de l'anatomie comparée, qui montre une grande analogie entre l'homme et les animaux, et plus particulièrement les singes, y compris dans la constitution du cerveau. Mais, ajoutait Gratiolet, le développement du cerveau de l'homme fait apparaitre les plis frontaux en premier lieu, les plis inférieurs en dernier, ce qui est l'ordre inverse du développement du singe. De sorte que «à aucune époque ce cerveau humain, semblable typiquement au cerveau du singe, n'est un cerveau de singe " ${ }^{11}$. L'homme, même du point de vue matériel, est complètement à part des êtres qui lui sont le plus semblables. Et si l'homme est un animal, "c'est un animal transfiguré, et si l'on peut ainsi parler, changé en symbole ". De telle sorte que, tout en ayant un langage commun avec celui des animaux, qui exprime chez chacun ses émotions et sa nature propre, et à travers lequel l'homme comme les animaux "se raconte", l'homme a un autre langage, le langage articulé et abstrait, " et ce langage n'appartient qu'à lui ». Grâce à cet autre langage, " l'homme ne se raconte pas seulement lui-même, il raconte les idées qu'il a de l'univers : aucun animal n'offre le moindre germe d'une pareille faculté $"$.

Au total, l'homme, par son organisation, est bien un animal : "c'est l'animal que traite le médecin. " Mais tout dans l'homme ne résulte pas de l'organisation. Des facultés toutes nouvelles se manifestent en lui, dont il n'y a même pas des ébauches chez le singe. Et Gratiolet en tire la question suivante : " loin de rapprocher l'homme du singe, leur similitude matérielle ne fait-elle pas mieux ressortir encore la profondeur de l'abîme qui les sépare?"

C'est sur un tel abîme que Gratiolet construit une réflexion qui fait de l'animal non un ancêtre, mais un modèle analogique de l'homme.

\footnotetext{
là les organes d'un langage universel. Elles reçoivent de cette haute destinée une importance irrécusable, supérieure peut-être à celle du mécanisme intérieur qui semble leur être subordonné dans l'harmonie de la corrélation réciproque des organes. »

10. P. Gratioler, "De l'Homme et de sa place dans la création " (Conférence dans le cadre des "soirées scientifiques de la Sorbonne "), Revue des cours scientifiques, 16, 19 mars 1864, p. 189-193 (sous la plume de Émile Alglave). Pour un commentaire de cette soirée, cf. Ernest Menault, "Leçon de Monsieur Gratiolet ", Le Moniteur scientifique-Quesneville, Paris, 1864 , p. $310-312$.

11. P. Gramolet, Mémoire sur les plis cérébraux de l'Homme et des Primatès (sic), Paris, Arthus Bertrand, 1854.
} 
Ainsi Gratiolet, spécialiste du cerveau et du système nerveux, articule d'emblée sa compétence sur un intérêt très vif pour tout ce qui concerne les mécanismes de l'intelligence, l'étude comparative du comportement animal et du comportement humain, en un mot, pour ce qu'il appelle luimême la psychologie:

«[...] certaines facultés sont communes aux animaux et à l'homme; ils ne diffèrent donc pas d'une manière universelle; mais d'un autre côté certaines facultés de l'homme n'appartiennent qu'à lui et ces facultés sont d'un ordre si relevé qu'elles font du genre humain un Règne à part dans l'armée des vivants. Je me suis donc attaché à les caractériser dans une esquisse rapide, mais ferme et précise, et en cela j'ai suivi la méthode des naturalistes plutôt que celle des idéologistes, mais si l'on juge que j'y ai réussi, peut-être paraîtrai-je avoir à mon tour payé mon tribut à la noble science de la Psychologie. "

C'est sur ces mots que Gratiolet termine sa préface du deuxième volume de L'Anatomie comparée du système nerveux. Dans cet ouvrage, il consacre en effet trois chapitres entiers à des problèmes de type plus psychologique qu'anatomique : le chapitre viI traite « Des effets de l'imagination ", le chapitre $\mathrm{Ix}$, de l'« Influence des mouvements extérieurs et des attitudes du corps sur l'imagination », et le chapitre $x$, « Des instincts et de l'intelligence ». On y trouve déjà beaucoup des conceptions sur les mouvements d'expression telles qu'elles sont développées dans l'ouvrage publié chez Hetzel. Mais ce qui prenait la place de quelques chapitres dans un ouvrage centré sur l'anatomie comparée, finit par devenir un objet central d'étude, où la physiologie et l'anatomie ne sont plus que des moyens pour comprendre la psychologie.

\section{LES CONCEPTIONS DE HETZEL SUR LA VULGARISATION :}

UNE SCIENCE AIMABLE ET SOLIDE

Le volume de Hetzel, regroupant la conférence de 1865 et l'ouvrage intitulé De la physionomie et des mouvements d'expression, fait se côtoyer en quelque sorte deux types de discours, le discours scientifique et le discours de vulgarisation. Il sera aisé, pour cerner les effets de vulgarisation, de confronter les deux textes que nous avons à notre disposition. 
Mais avant même d'entrer dans les textes de Gratiolet, il n'est pas inutile de s'attarder sur les propos préliminaires de l'éditeur, c'est-à-dire Hetzel lui-même, qui, à travers l'éloge de la conférence, définit pour ainsi dire les conditions de la parfaite vulgarisation. On sait que, pour Hetzel, la vulgarisation scientifique est une sorte de mission humanitaire et fait l'objet d'une véritable passion. L'éditeur du Magasin d'éducation et de récréation, créé en 1864 , et auquel Gratiolet participe comme rédacteur de la partie " éducation " ${ }^{12}$, est donc très sensible au talent oratoire et au sérieux scientifique.

Dans sa préface, Hetzel commence par rappeler l'ancienneté et l'universalité de l'intérêt pour la physionomie, ainsi que ses enjeux dans les relations sociales, toutes choses qui contribuent à faire du geste signifiant un objet qui intéresse le grand nombre. Intérêt plein d'embûches, cependant, car l'impatience, le désir d'appliquer immédiatement des résultats encore fragiles, en un mot cet «attrait du merveilleux » a donné naissance à bien des discours qui sont « à la vraie physiognomonique ce qu'est à l'astronomie la science des almanachs » et Hetzel rappelle la crainte de Gratiolet de se voir confondu « avec ces bas imitateurs de Lavater " ${ }^{13}$. Mais, dit Hetzel, Gratiolet a su échapper à tous ces dangers, et a réussi à construire un discours qui soit attrayant sans cesser d'être rigoureux. À travers les traits où, selon Hetzel, Gratiolet a excellé, on voit se profiler les qualités que l'on peut attendre de la vulgarisation scientifique.

La séduction d'abord est soulignée comme dimension indispensable : en effet, la conférence du 20 janvier a été " accueillie par des acclamations enthousiastes $»^{14}$. Mais les qualités internes de l'exposé ne sont pas moins importantes : une thèse clairement résumée, dépouillée de dogmatisme, une alliance de rigueur et de poésie, qui a fait de cette conférence " un véritable chef-d'œuvre de science aimable et solide", dont la lecture est " entrainante comme celle d'un roman " 15 .

12. Cf. Denise Dupont-Escarprt, « L'information scientifique et technique à l'usage de la jeunesse dans Le Magazin d'éducation et de récréation », in Un Éditeur et son siècle, PierreJules Hetzel (1814-1886), Saint-Sébastien, A.C.L. Éditions, 1988, p. 237-248. Sur la présence de Gratiolet parmi les rédacteurs du Magasin d'éducation et de récréation, cf. Ségolène LE MEN, "Hetzel ou la science récréative », Romantisme, Science pour tous, 65, Paris, C.D.U./Sedes, 1989 , p. $69-81$.

13. Cf. P. Gratiolet, op. cit. supra n. 1 d), p. II-III.

14. Ibid., p. IV.

15. Ibid., p. IV-V. 


\section{LA PROPORTION DES ANALOGIES DANS LA RECHERCHE}

ET DANS LA VULGARISATION

Si l'on considère la conférence de Gratiolet, on y voit apparaitre plusieurs types de procédés de vulgarisation, comme le recours à l'intuition de l'auditoire et à l'expérience personnelle ${ }^{16}$, et surtout cette utilisation d'images et d'analogies avec le monde animal.

On pourrait s'attendre à ce que ces analogies soient proportionnellement plus fréquentes dans la conférence que dans le texte de recherche. Or il n'en est rien. Sur les 68 pages de la conférence, 8 sont consacrées à des analogies animales, soit environ 1/9. Dans le texte de recherche qui compte 315 pages, 50 sont consacrées à de telles analogies, soit environ 1/6. Cela tient au fait que la conférence utilise aussi d'autres procédés de vulgarisation. Mais l'importance de ces analogies dans le texte de recherche laisse supposer que le recours à l'animal n'est pas pour Gratiolet un simple effet de vulgarisation, mais a bien une fonction scientifique. Comme on a vu que la perspective de Gratiolet interdit une interprétation en termes transformistes, et ne peut faire du geste animal un geste originaire pour l'homme, il convient de faire apparaître les fonctions de l'analogie animale et de voir si elles sont les mêmes dans la conférence et dans le texte de recherche.

\section{LES FONCTIONS DE L'ANALOGIE ANIMALE DANS LA CONFERENCE :}

\section{1) Le comportement humain comme comportement de substitution.}

Tout le système d'explication du comportement signifiant repose chez Gratiolet sur une distinction, qu'il a mise en place depuis avant 1853, entre quatre types de mouvements : les mouvements directs, les mouvements sympathiques, les mouvements symboliques et les mouvements métaphoriques. Â ces types de mouvements, à l'exception du troisième ${ }^{17}$, correspondent des fonctions particulières du recours à l'analogie animale.

16. Cf. P. Granolet, op. cit. supra n. 1 d), p. 2 : « Il me suffira de faire appel à la connaissance que vous avez de vous-mêmes. C'est en vous, c'est dans vos souvenirs et dans vos sentiments intimes que je trouverai mes preuves ; c'est dans votre raison seule que j'espère trouver la justification de mes paroles. "

17. Cf. P. Gratiolet, op. cit. supra n. 2, p. 631-639 : Les mouvements symboliques sont les mouvements éveillés par l'imagination, qui font que si l'on pense à une émotion ou une sensation, le corps va réagir comme si elle était présente. Imaginer qu'on tremble fait trembler... Mais de fait, l'explication des mouvements symboliques est peu éclairée par l'analogie avec les animaux, Il apparaît, en effet, dans l'Anatomie comparée du système nerveux, que Gratio- 
Dans la conférence, la première occurrence de l'analogie animale a pour but de montrer que l'animal est particulièrement bien doté d'organes faits pour réagir à certaines situations et que, par contraste, l'homme en est moins bien pourvu, ce qui génère des comportements de substitution. Ce premier type d'analogies correspond au premier type de mouvements. Comme leur nom l'indique ces mouvements ont un but direct, immédiatement interprétable : c'est l'œil s'ouvrant sur ce qu'il regarde, l'oreille se tendant vers ce qu'elle écoute, ou inversement l'œil comme l'oreille se détournant d'un objet pour lequel existe une aversion. C'est en quelque sorte un degré zéro de la signification, où la distance entre le signe et ce qu'il signifie est quasi nulle.

Or, chez l'homme, certains organes étant moins bien développés que chez les animaux, certaines attitudes semblent incompréhensibles. L'analogie animale vient alors comme une traduction. Un exemple sur lequel Gratiolet s'attarde un peu est celui du regard de la peur. Dans ce regard, les yeux semblent vouloir fuir en arrière. Il suffit de considérer le comportement d'un animal comme le lapin ou le lièvre pour rendre intelligible cette tendance du regard:

" [...] ce regard est très facile chez certains animaux timides, chez les lièvres et les lapins par exemple, dont les yeux situés aux deux extrémités d'un diamètre transversal de la tête ont une égale facilité à voir en avant et en arrière du corps; cette facilité leur est fort précieuse, car sans cesse exposés aux attaques des animaux carnassiers, ils peuvent ainsi, dans leur fuite éperdue, échapper plus aisément au danger qui les menace en mesurant constamment la distance qui les sépare encore du renard, du loup ou du chien qui les poursuit, sans avoir à tourner la tête [...] Le parallélisme des axes oculaires rend chez l'homme ce regard en arrière absolument impossible. Il est cependant certains cas [...] où les yeux ont une tendance évidente, bien qu'inutile, à regarder ainsi; on les voit alors simultanément se porter d'un côté ou de l'autre, jusqu'aux limites extrêmes de ce mouvement, et l'on dirait, passezmoi cette expression, qu'ils veulent faire le tour de la tête $"{ }^{18}$.

À propos du phénomène de l'audition et de l'écoute, le geste humain est décrit là encore comme un geste de substitution. Son oreille n'étant pas munie, comme celle des chiens et des chevaux, d'un pavillon mobile,

let, bien qu'il accorde l'intelligence aux animaux, ne leur accorde pas le pouvoir d'avoir des images mentales fortes au point d'avoir comme chez l'homme une quasi-réalité objective. Néanmoins, dans ce même ouvrage, il utilise le modèle animal pour éclairer le phénomène inverse des mouvements symboliques, à savoir l'influence du corps sur l'imagination. Cf. A.M. Droun, " Le modèle animal dans l'explication du comportement humain chez Pierre Gratiolet (1815-1865) ", $115^{\circ}$ Congrès des sociẻtés savantes, Avignon, 1990, Paris, Éd. du Comité des travaux historiques et scientifiques, 1991, p. 123-136.

18. Cf. P. Gratiolet, op. cit. supra n. 1 d), p. 16-17. 
l'homme doit incliner le cou, au point parfois de se tendre avec effort dans la direction de la source sonore et, dans ce cas, le visage est crispé, la bouche forme un rictus, des rides se forment sur la joue... " on dirait en un mot que tous les muscles de la face s'efforcent de suppléer à l'insuffisance des muscles du pavillon $" 19$.

\section{2) Des mouvements amplifiés et plus lisibles chez les animaux.}

Un usage des analogies apparaît à propos de certains mouvements des animaux, qui ont une amplification par rapport aux mouvements humains équivalents et qui, selon Gratiolet, les rend plus facilement interprétables.

C'est le cas des mouvements directs des organes des « sens inférieurs", c'est-à-dire l'odorat et le goût. On les voit particulièrement à l'œuvre chez les carnassiers chasseurs, dont le nez est décrit comme « un directeur du corps tout entier". Chez l'homme, "ces mouvements sont à la vérité moins prononcés ", dit Gratiolet, mais il n'est pas moins certain " qu'une odeur agréable attire la tête, et qu'une odeur mauvaise la repousse $"{ }^{20}$. Ce qui est à peine perceptible dans l'attitude humaine, devient alors visible grâce au recours à l'exemple animal.

C'est aussi le cas pour la deuxième catégorie de mouvements de Gratiolet : les mouvements sympathiques. Ce terme désigne les mouvements des parties du corps non directement concernées par une certaine sensation, mais qui s'associent comme par sympathie avec l'organe concerné : "Un son désagréable déchire-t-il les oreilles, les yeux se ferment d'euxmêmes", disait-il dans l'Anatomie comparée du système nerveux ${ }^{21}$.

C'est ce qu'il nomme dans sa conférence la «république des organes ${ }^{22}$. Cette participation de tous les organes à un organe qui reçoit une sensation est illustrée par la description toute poétique d'un petit chat devant un bol de lait sucré :

"Voyez-le s'avancer lentement et flairer avec attention; ses oreilles se dressent, ses yeux largement ouverts expriment le désir, sa langue impatiente léchant les lèvres, caresse et déguste d'avance l'objet désiré. Il marche avec précaution le cou tendu. Mais il s'est emparé du liquide embaumé, les lèvres le touchent, il le savoure; !'objet n'est plus désiré, il est possédé; le sentiment que cet objet éveille s'est emparé de l'organisme tout entier; le petit chat ferme alors les yeux, se considérant lui-même tout pénétré de plaisir. Il se ramasse de lui-même, il fait le gros dos, il frémit voluptueusement, il semble envelopper de ses membres, son corps, source de jouissances ado-

19. Ibid., p. 19-20.

20. Ibid., p. 22.

21. Cf. P. Granolet, op. cit. supra n. 2, p. 658.

22. ID., op. cit. supra n. 1 d), p. 33. 
rées, comme pour le mieux posséder; sa tête se retire doucement entre ses deux épaules, on sent qu'il cherche à oublier le monde, désormais indifférent pour lui; il s'est fait odeur, il s'est fait saveur, et il se referme en lui-même avec une componction toute significative ${ }^{23}$.

Symétriquement, la douleur peut aussi produire ces réactions synthétiques de tous les organes. Mais la douleur est source de réactions contradictoires : quand elle est profonde, c'est le repli sur soi, les yeux fermés; quand elle est violente, les yeux sont grands ouverts et hagards. Les deux attitudes trouvent leur intelligibilité grâce à l'analogie animale.

Dans le premier type de douleur, on retrouve l'animal « qui se retire dans quelque endroit écarté », et qui "recherche les ténèbres et le silence $"{ }^{24}$. Dans le deuxième, Gratiolet appuie son propos par l'analogie du chat qui s'est brûlé la patte et évoque ainsi cette forme extrême de la douleur, dans laquelle les mouvements désordonnés des membres semblent vouloir arracher le mal, voire même arracher la partie du corps qui est le lieu de la douleur ${ }^{25}$.

3) Chez les animaux, des métaphores plus grossières et plus facilement lisibles.

Pour Gratiolet, « le geste de l'homme est plein de métaphores, et instinctivement les animaux en font aussi quelques-unes $"{ }^{26}$. Ainsi, le refus se marque par un resserrement du nez et de la bouche comme pour écarter l'objet refusé, le plaisir extrême, par une ouverture des narines, comme ces lecteurs délicats qui semblent " par leurs mouvements, à la lecture de certains passages, s'enivrer d'un parfum céleste ${ }^{27}$

« D'un homme qui lit ainsi, ajoute Gratiolet, vous direz naturellement : c'est un homme de goût. [...] D'un homme qui plaît dans le monde on dit métaphoriquement qu'il est goûté. [...] N'est-ce pas une preuve entre mille que les métaphores du geste sont parallèles aux métaphores du langage? ${ }^{28}$.

Ces mouvements métaphoriques, qui forment la quatrième catégorie de mouvements, sont illustrés d'exemples pris dans le monde animal, bien que les animaux soient moins portés à la métaphore que les hommes. Il faut voir deux raisons à cela : d'une part, Gratiolet peut montrer à quel point les métaphores du geste existent d'abord spontanément, et non

\footnotetext{
23. Ibid., p. 31 .

24. Ibid., p. 33

25. Ibid., p. 33

26. Ibid., p. 41 .

27. Ibid., p. 45 .

28. Ibid., p. 45-46.
} 
conventionnellement; et, d'autre part, ces métaphores étant plus grossières chez les animaux que chez l'homme, elles sont aussi plus facilement lisibles.

Si l'on prend la bienveillance par exemple, accompagnée de la joie et de l'amour, chez l'homme elles se manifestent ainsi :

" [...] les lèvres agitées par de petits mouvements de dégustation agréable, les mains toujours prêtes à recevoir, à serrer doucement, à caresser, et enfin le baiser, cette caresse des lèvres qui semble attirer symboliquement l'âme de l'être aimé ${ }^{29}$.

Chez un animal comme le chien, on a quelque chose de proche, mais de plus grossier :

" Parmi les animaux les chiens seuls sont capables d'exprimer avec une évidente clarté l'amour et la bienveillance. Ils lèchent en agitant la queue ceux qu'ils aiment, ils les contemplent de leurs yeux ardemment fixés, ils aboient pour solliciter le regard; ils éveillent par de petits coups de leurs pattes antérieures l'attention de ceux qu'ils aiment : rien n'est plus éloquent ${ }^{30}$.

Une note en bas de page fait le point sur la différence entre les métaphores chez l'homme et l'animal :

"C'est là une expression analogue au baiser de l'homme; mais le baiser est un mouvement de la bouche considéree comme organe respiratoire. Le chien lèche ; et cette forme, empruntée à la bouche en tant qu'elle est un organe de la vie nutritive, est évidemment inférieure ${ }^{31}$.

Embrasser, pour l'homme, c'est en quelque sorte respirer l'autre; pour le chien, lécher c'est presque ingérer. On va ainsi de métaphore en métaphore, jusqu'à la grossièreté des " fonctions inférieures », qui expriment si bien pourquoi aimer c'est aussi goûter.

4) Rendre visible ce qui était invisible : le mouvement direct de l'animal comme métaphore pour l'homme

Il existe, outre les exemples d'expressions simples, des expressions " mixtes " qui ont pour particularité de pouvoir renfermer des sentiments contradictoires. Là encore, Gratiolet a recours à l'analogie animale, mais en procédant à quelques détours. Ce qui chez l'homme est pris comme métaphore, était simple mouvement direct chez l'animal : l'animal agit

29. Ibid., p. 46.

30. Ibid., p. $47-48$.

31. Ibid., p. 48. 
directement, et l'homme fait en quelque sorte comme si. Par exemple, pour décrire l'incertitude chez l'homme, Gratiolet passe par la description d'« un chien affamé à qui l'on présente une pâtée savoureuse » :

"Il se précipite sur elle, mais elle est bouillante; à peine y a-t-il touché qu'il recule subitement; mais à mesure qu'il s'éloigne, l'impression et la crainte de la douleur s'effaçant, le désir se réveille " ${ }^{32}$.

À travers cet exemple, les caractéristiques de l'hésitation, à savoir les mouvements alternatifs du corps, sont mises en évidence. Les mouvements de va-et-vient du chien « traduisent dans un sens métaphorique les incertitudes de l'esprit dans des circonstances toutes morales ${ }^{33}$. Et ainsi, lorsque Gratiolet en vient à l'homme lui-même, sa description prend alors un relief étonnant :

«Au bout de quelques instants il ne tient plus en place, un lutin capricieux le tourmente. Était-il couché ? Il se lève. Levé, il se recouche; il se tourne sans cesse de gauche à droite et réciproquement. Tantôt il étend ses jambes, tantôt il les replie $[\ldots]{ }^{34}$.

Dans ce mouvement où le comportement humain se calque métaphoriquement sur celui de l'animal, l'analogie rend pour ainsi dire visible ce qui était invisible.

Après avoir vu ces fonctions explicatives de l'analogie animale, on peut se demander si elles sont réservées à la vulgarisation ou si elles ont une fonction scientifique.

LES REFERENCES AUX ANIMAUX DANS LE TEXTE SCIENTIFIQUE

\section{1) Les principes de l'anatomie comparée.}

Dans le texte qui a pour titre De la physionomie et des mouvements d'expression, Gratiolet se centre sur les diverses categories de mouvements, et garde assez longtemps un point de vue plus physiologique et expérimental que sémiologique. En fait, les données physiologiques apparaissent comme le préalable nécessaire à la compréhension des gestes signifiants, et la perspective sémiologique se développe comme un prolongement de la physiologie.

32. Ibid., p. 56-57.

33. Ibid., p. 57.

34. Ibid., p. 58. 
Il évoque souvent les animaux, et sa perspective est celle de l'anatomie comparée. La confrontation de la physiologie animale et de la physiologie humaine permet un éclairage pertinent sur certaines manifestations expressives : sur le rire nasal par exemple ${ }^{35}$, qui n'est pas propre à l'homme, puisqu'on le retrouve dans les espèces du genre equus, sur les manifestations de l'effort chez les animaux à sang chaud ${ }^{36}$, sur la description des attitudes du bâillement chez certains mammifères ${ }^{37}$, etc.

\section{2) Des descriptions plus brèves.}

Toutes ces attitudes sont lues à travers les mécanismes physiologiques qui, par leur logique explicative, sont pour le corps de véritables signes à interpréter. Et, comme on l'a vu, c'est précisément parce que l'interprétation est plus directement lisible chez les animaux que Gratiolet s'y réfère souvent. On retrouve ainsi dans De la physionomie certaines des descriptions développées dans la conférence. Mais la description, est beaucoup plus brève et plus sobre. Par exemple, celle de l'œil des « animaux timides " :

« Cette disposition [celle consistant à mettre les deux yeux vers l'arrière] est fort habituelle aux animaux timides, auxquels il importe de surveiller l'ennemi pendant la fuite afin de mieux éviter ses atteintes ${ }^{38}$.

Dans cet exemple, Gratiolet ne s'attarde pas particulièrement sur l'homme, qui est implicitement inclus dans l'ensemble des animaux dont les orbites ont leurs axes à peu près parallèles, et qui pour suppléer à cette gêne voient leurs deux yeux se porter alternativement à droite et à gauche $^{39}$. De même la description de l'audition chez les animaux qui n'ont pas d'oreille externe mobile permet d'inclure l'homme dans certaines de ses attitudes, mais là encore de façon assez brève :

« Et dans l'impossibilité où l'animal se trouve de rapprocher à la fois du même point les deux côtés de la tête, il n'écoute que d'une seule oreille à la fois, mais il les emploie alternativement l'une et l'autre, soit pour prévenir la fatigue d'une seule oreille, soit pour les faire participer toutes les deux à de douces impressions. Ce mouvement est bien marqué chez l'homme et chez certains animaux que la musique charme, tels que beaucoup d'oiseaux et de sauriens et peut-être même quelques ophidiens $"{ }^{40}$.

35. Ibid., p. 114.

36. Ibid., p. 119-120.

37. Ibid., p. 126.

38. Ibid., p. 147.

39. Ibid., p. 148.

40. Ibid., p. 150-151. 
Enfin, l'analogie du chien qui se brûle, pour expliquer les manifestations de l'hésitation, est présente sous une forme plus neutre :

« L'hésitation est une sorte d'oscillation entre le désir et la crainte. Les jeunes chiens nous en donnent un exemple curieux, lorsqu'on leur présente un mets dont l'odeur les allèche mais dont la chaleur les blesse. Au moment où ils touchent à l'objet ils sont brûlés et reculent. Puis l'impression de brûlure cessant, l'odeur les sollicite et ils sont attirés de nouveau. Ils oscillent ainsi, et des mouvements d'impatience se mêlant à ces oscillations, il en résulte l'une des expressions les plus puissantes. Ces phénomènes ne sont pas moins apparents dans l'espèce humaine. Mais alors l'oscillation peut se produire non seulement entre deux sensations mais si je puis ainsi dire entre deux idées $\gg{ }^{41}$.

\section{3) De l'analogie au modèle}

Ainsi l'ouvrage théorique, loin de dédaigner l'analogie animale, lui donne en quelque sorte un véritable statut de modèle. Les exemples précédents sont significatifs à cet égard. Sur le contenu, ils offrent peu de différence avec les propos de la conférence. Mais l'éclairage de l'anatomie comparée tend à leur retirer tout caractère fantaisiste. Et d'autres exemples, non présents dans la conférence, confirment ce rôle de l'animal comme modèle. Par exemple, dans sa description de l'attention extrême chez l'homme, Gratiolet dit qu'il est nécessaire que le corps soit d'une immobilité parfaite; or, en tant que bipède l'homme a de grandes difficultés à obtenir cette immobilité ; c'est pourquoi il appuie ses mains sur ses genoux afin de trouver «une véritable station quadrupède oblique ${ }^{42}$.

Le recours à l'animal peut aussi faire fonction de modèle inversé, lorsque, à propos de l'usage de la langue comme organe de préhension (chez la girafe, par exemple), Gratiolet souligne à quel point cet usage est proscrit dans le monde humain, non pas tant pour des raisons physiologiques que pour des raisons culturelles :

«On retrouve dans l'homme enfant des mouvements analogues [à ceux de la langue de la girafe]. Mais comme ils rappellent la voracité brutale des bêtes, les hommes chez lesquels le sentiment de la moralité s'élève en proscrivent l'emploi $"{ }^{43}$.

41. Ibid., p. 386.

42. Ce propos est illustré par un dessin. Cf. P. Gratiolet, op. cit. supra n. 1 d), p. 223.

43. Ibid., p. 157-158. 
Pour résumer les diverses fonctions que Gratiolet fait jouer aux références animales, tant dans la conférence que dans le texte scientifique, on peut retenir que l'animal sert à mieux cerner le but d'un comportement, à mieux voir la structure du mouvement lui-même, à rendre sous forme imagée ce qui était surtout mental, à cerner le signe dans son essence même. Décrire l'animal, c'est comprendre l'homme.

Mais si l'on peut parler de modèle dans tous ces exemples, c'est que l'animal y est néanmoins montré dans sa différence : l'analogie souligne aussi la distance. Les comportements animaux ne sont vus ni comme identiques à ceux des hommes, ni comme leur genèse. Le recours à l'animal apparaît comme un détour théorique, qui a pour fonction de rendre visible ce qui ne l'est pas et explicite ce qui est implicite. L'analogie est un processus de concrétisation, et une mise en forme cohérente de ce qui restait chaotique. Dans tous les cas, il s'agit de produire un gain de sens, par des outils intellectuels dont on garde la maitrise, sans confusion entre l'image et ce dont elle est image : ces caractéristiques sont bien celles que l'on attribue d'ordinaire aux modèles.

DE LA VULGARISATION À LA SCIENCE?

La comparaison entre le texte de vulgarisation et le texte scientifique tend ici à réduire les différences entre les deux discours. Il reste que sur le plan du style, la conférence donne plus de place à des descriptions vivantes, comme de véritables petites scènes de genre. Mais précisément, dans ce changement de style vient peut-être se glisser un changement de statut de la référence à l'animal.

En effet, si le comportement humain devient lisible grâce au comportement animal inversement, dans la conférence, les comportements des animaux, sont eux-mêmes bien souvent interprétés en fonction d'un modèle humain. Voulant rendre ses tableaux vivants, Gratiolet prête aux animaux des désirs, des craintes, des intentions, des stratégies, calqués sur les comportements humains. L'explication est prise alors dans un cercle vicieux. Les précautions méthodologiques et la prudence interprétative sont estompées au profit de la volonté de faire comprendre à tout prix et du plaisir de la poésie.

Pourtant, il s'agit pour Gratiolet de lire les gestes comme des signes, et de faire une étude comparative entre l'homme et les animaux, étude dont l'objet est de mettre en évidence la spécificité du comportement humain. 
Les propos de Gratiolet, vus sous cet angle, semblent bien participer à l'élaboration d'une science du comportement.

Et paradoxalement, si l'on considère que ce domaine de recherche, à savoir la sémiologie du geste, doit, pour se constituer en discipline autonome, s'affranchir d'une trop étroite affiliation à la physiologie, la genèse de cette discipline nouvelle est sans doute plus présente dans la conférence que dans le texte scientifique. En effet, dans la conférence le point de vue est d'emblée sémiologique. Gratiolet commence par y affirmer l'existence d'un langage universel, commun aux hommes et aux animaux, celui de la physionomie et du geste, qui « est parlé dès le commencement des choses ${ }^{44}$. Ce langage $«$ a ses règles simples et intelligibles ${ }^{45}$, dont la logique repose, comme on l'a vu, sur le fonctionnement de la sensibilité et des mouvements. Ceux-ci, même s'ils ne sont pas par nature destinés à l'expression, fournissent des indications sur l'état d'esprit des individus, et deviennent des signes à interpréter ${ }^{46}$. Il expose par ailleurs un résumé assez détaillé et critique des études qui ont précédé la sienne depuis l'Antiquité sur l'étude de la physionomie. Il précise son apport personnel face à ses prédécesseurs. Bref, il se pose en initiateur d'une science nouvelle. Tout se passe comme si, par une sorte de ruse de la Raison scientifique, les efforts de vulgarisation avaient pour effet non pas tant de transposer en un discours plus simple des contenus scientifiques compliqués, mais bien d'inventer un discours nouveau. Dans cet exemple particulier, ce ne serait plus la vulgarisation qui découlerait de la science mais la science qui découlerait de la vulgarisation. Et dans ce flou des frontières entre science et vulgarisation, caractéristique d'une discipline en train de naître, on observe comment, dans sa genèse, une discipline oscille entre l'analogie, les efforts de formalisation et les exigences de vérification.

Anne-Marie Drouin-Hans, Université de Bourgogne, Dijon.

44. Ibid., p. 1-2.

45. Ibid., p. 12.

46. Cf. ibid., p. 12-13: «[...] il n’y a pas un seul muscle, un seul organe créé uniquement pour les besoins de l'expression. Tout organe, en effet, a en principe un but extérieur, un but déterminé. Ce but il le raconte par sa forme, et par son activité propre; or [...] le degré d'énergie d'un mouvement quelconque fournit des indications immédiates. " 